\title{
Network size variation of geographical aided routing protocols
} in MANET

\author{
Radhwan B. Al-Bayram ${ }^{1}$, Radhwan M. Abdullah ${ }^{2}$ \\ ${ }^{1}$ Department of Computer Sciences, College of Computer Sciences and Mathematics, University of Mosul, Mosul, Iraq \\ ${ }^{2}$ Division of Basic Sciences, College of Agriculture and Forestry, University of Mosul, Mosul, Iraq
}

\begin{tabular}{|c|c|}
\hline Article Info & ABSTRACT \\
\hline Article history: & \multirow{9}{*}{$\begin{array}{l}\text { In position-based or geo-casting ad hoc networking applications, the data } \\
\text { packets flooded in a limited area of the expected region toward the } \\
\text { destination node. In such networks, a multi-hop routing is to followed when } \\
\text { the position of the destination node is residing off-range the wireless } \\
\text { transmission of the source node. Scientific literature references in this } \\
\text { concept have been conducted depended on the diversity of quantitative } \\
\text { metrics-tests regarding the size of the assigned networks so that this space is } \\
\text { intended to be the variation in the density of the moving nodes (the number } \\
\text { of nodes per unit of measurement). In this work, we studied the impact of the } \\
\text { change in the size (area) of the ad hoc network with the concept of variation } \\
\text { in the network's area while remaining the total number of nodes as a constant } \\
\text { volume. In this paper, three of the position-based routing protocols have } \\
\text { evaluated in terms of a network size variation. These routing protocols are: } \\
\text { locations aided routing LAR, the distance routing effect algorithm for } \\
\text { mobility DREAM, and the greedy perimeter stateless routing GPSR. With } \\
\text { each simulation test, a quantitative metrics evaluation determined the most } \\
\text { efficient protocol and the most efficient accessibility with the network } \\
\text { environment changes in terms of actual workspace. }\end{array}$} \\
\hline Received Mar 20, 2020 & \\
\hline Revised Jun 26, 2020 & \\
\hline Accepted Jul 28, 2020 & \\
\hline Keywords: & \\
\hline Ad hoc & \\
\hline NS-2 simulation & \\
\hline Position-based & \\
\hline Scalability & \\
\hline
\end{tabular}

This is an open access article under the CC BY-SA license.

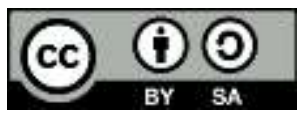

Corresponding Author:

Department of Computer Sciences

College of Computer Sciences and Mathematics

University of Mosul, Mosul, Iraq

Email: radhwanbasher@gmail.com

\section{INTRODUCTION}

Ad hoc networks are types of unconventional wireless networks, wherein traditional wireless networks, the communications between the nodes in the networks are done under full administration's control of central distributing communication applications (Ex., Wi-Fi LANs and Cellular Networks communications). On another side, with the absence of a central-fixed point of administrating and with nodes movement in dynamical behavior; this distributed communication can be categorized as "Mobile Ad hoc Network or MANET".

MANET applications in the last few years get a great focusing from the world community deal with modern-smart information technology and IoT supporting techniques. This came from the power of the scalability and other characteristics feature supported by MANET, where these networks, for example, but not limited to, can be established at "anytime and anywhere" as a short-lived and quickly installation system with the capability of propagation and toleration this expansion. Moreover, these networks can modulate the increasing of the average number of mobile devices that should be serviced. All this with maintaining as much as possible the critical resources in the network such as bandwidth consumptions, computation 
processing overheads, and nodes energy. These days, ad hoc protocols have been embedded as routing protocols in many cutting-edge projects like Vehicle Ad hoc Networks VANET, Flying Ad hoc Networks FANET, and Wireless Sensor Networks WSN [1].

In common, the classifications of mobile ad hoc routing protocols divided into two main types; topology (flat) routing based, and position (geographic) routing based. Furthermore, each mentioned type can be subdivided into other types, i.e. (proactive, reactive, and hybrid), as illustrated in Table 1 [2].

Table 1. Common classification of ad hoc routing protocols

\begin{tabular}{lll}
\hline & \multicolumn{1}{c}{ Topology type } & \multicolumn{1}{c}{ Position type } \\
\hline Proactive & OLSR, FSLS, DSDV, TBRPF [3-6] & DREAM, DRM, REGR [7-9] \\
& DSR, AODV, LMR, TORA [10-13] & LAR, Geo-TORA, ALARM [14-16] \\
Hybrid & ZRP, OORP, HSLS, ZHLS [17-20] & GPSR, OGPR [21, 22] \\
\hline
\end{tabular}

The proactive routings in MANET have a distributed system where every mobile device (node) at any time-has a buffered space of routes to some or all other devices in the network. In fact, the pros of this type can summarized in scientifically reducing of the routing overhead since there no need to route discovery phase as long as every node broadcast its routing table periodically to all nodes in the network, so each node has up-to-date information about others in the network, while the cons of this type are consists of needing to more bandwidth for maintenance the updating information that sends in fixed period time and the wasted cost in updating idle nodes.

In proactive routing algorithms, the overhead computations of routing are scientifically reduced by controlling request packets on demands. When any node needs to send a data, its first start looks in the routing table if there is an existing route to the destination, then, it sends the data-packed with piggybacked the routing path, else, it starts to initiate the request packet. The route requests are maintained by the intermediate node until reaching the destination node which acknowledged back with rout replay packet [23].

The previous routing types (proactive and reactive) serviced as routing supporting ideas of MANETs; while another side of classification could depend on the underneath topology structure to followed in flooding the forwarded data packets in the space whole or maybe in a specific area of the network, this could lead to present two methods of routing approaches: flat type and position-based type. The routings in flat topology type are considered all nodes in the network as one level of function and the routing is adapted the flat addressing form where the decision initiated in source node to find the best route (shorted path) to the destination is dependent on-generally-to distance-state or link-state schemes. The demonstration of the full picture in the network in flat type routing proceeds a short-time for delivering the data packets but with the side effect in routing throughputs throughout the increasing of the routing overhead, especially in high dynamic nodes and network area extension.

In position aided routing type and to overcome the drawbacks in flat topology type, the flooding data can be sent to a limited geographic area in the network with the assistant of GPS technologies. The nodes in this type of routings are initially assumed to know their positions and then employed the location service methods to locate the position of desire partner. A survey of position-based protocols and location service methods can be found in $[2,24]$ respectively. In concentrating on the factor of scalability which is mean how the distributed system can be tolerant with increasing of nodes number or even the network area itself, we can consider the main scientific progression of position assistant routing types above the flat topology types in MANET is the scalability issue [25].

The aspect of scalability in ad hoc geo-casting routing protocols has addressed by a valuable number of articles in literature studies [26-29]. Most of these articles considered in analyzing the fact of scalability in MANET the extension of network size is associated with increasing the number of mobile nodes in the network area. While on another face of fact, it has not taken into consideration the geographical extension model as a function of a fixed number of a mobile node when verification of the scalability. In this work, we simulate three algorithms of position based ad hoc routing protocols in order to evaluate the performance for each method in term of the scalability factor with consist of a variation of network size in respective to a geographical area with keeping the total number of working nodes as a constant.

In Section 2 of this article, we present a literature reviewer of ad hoc position-based routing protocols in terms of scalability. In the 3rd section, we demonstrate and discuss three routings of position aided ad hoc methods. Simulation environments and evaluation of quantitative metrics discussed in Section 4. Section 5 presents the simulation results and discussion. Section 6 concludes this article. 


\section{SCALABILITY REVIEW IN POSITION- BASED AD HOC ROUTINGS}

In this section, we present some works have done in terms of scalability in position-based MANET protocols. Where on almost previous studies, they have focused on defining the scalability as the increasing of node's density with remaining the dimensions of geographical area as constant or in another definition the increasing of node's density will be accompanied by increasing of the network area, while herein the motivation of this research is to evaluate the mentioned ad hoc routing types in another definition of scalability which is "extensions the dimension's of network area with decreasing the ratio of node's density by remaining the volume of nodes unchanging".

Three types: flooding-based routings, routing-based protocols, and cluster-based routing algorithms, then selected one typical protocol from each category and performed a simulated evaluation of these protocols in four comparison studies. The network area size is fixed to $300 \mathrm{x} 600$ meters in all their evaluation studies and the first study "dense nodes" they variation the density in the static network area from 30 to 120 nodes.

In [27] the author presents comparisons between four ad hoc routing protocols (AODV, DSR, LAR, and ZRP). The author employed a simulation-based type and theoretical model to evaluate the scalability of these protocols. The scalability was performed by changing network size and nodes density. The simulations were considered five variations in network sizes area (from 1500x300 to 6708x1342) with remaining the ratio of node density as a uniform fashion for each geographical area. To return to our proposed method, we are assuming a constant number of nodes even with the changing in network size geographically.

The challenges in network scalability of large ad hoc networks with the irregularity of obstacles and holes area issues are addressed in [28]. The authors proposed a new technique called "Terminode routing". This technique is depending on putting pseudo anchored points in the routing path between the source node and destination. The terminode protocol has compared with location-based routing protocols (LAR and GPSR) and link-based routing protocols (AODV), the authors discussed and evaluated five experiential studies, four of them have been achieved by simulation results and one by theoretical computations. All simulation evaluations were depended on changing network area (2200x600 to 2900x2900) with remaining the node density of each issue as a fixed ratio number.

The paper in [29] evaluated the performance of DREAM routing protocol in a large network; (the number of mobile nodes has chosen from 50 to 500) using a so-called realistic mobility model RealMobgen [30], while the area of the network was stickered to $900 \mathrm{x} 1200$ meter. Table 2 summarizes the behavior of the trend used in previous reviewer articles in terms of the scalability evaluation in positionbased ad hoc routing protocols.

Table 2. Summary of reviewed articles regards the scalability factor

\begin{tabular}{|c|c|c|c|c|c|}
\hline Reference & Simulation & Routing Protocols & Network area(m) & Node no. & NDR* \\
\hline [26] & NS2 & $\begin{array}{l}\text { LBM, GAMER, } \\
\text { GeoGRID }\end{array}$ & single $300 \times 600$ & $30-120$ & --- \\
\hline [27] & $\begin{array}{l}\text { QualNet, } \\
\text { theoretical }\end{array}$ & $\begin{array}{l}\text { AOVD,DSR, } \\
\text { LAR,ZRP }\end{array}$ & $\begin{array}{l}1500 \times 300,2121 \times 424,3000 \times 600, \\
4743,949,6708 \times 1342\end{array}$ & $50-1000$ & uniform \\
\hline [28] & $\begin{array}{l}\text { GlomoSim } \\
\text { theoretical }\end{array}$ & $\begin{array}{l}\text { LAR, GPSR, } \\
\text { AODV }\end{array}$ & $\begin{array}{l}2200 \times 600,3000 \times 2500 \\
2900 \times 2900\end{array}$ & $\begin{array}{l}100,500 \\
600\end{array}$ & Irregular \\
\hline [29] & NS2 & DREAM & single $900 \times 1200$ & $50-500$ & --- \\
\hline
\end{tabular}

\section{POSITION-BASED AD HOC ROUTING PROTOCOLS}

In this section, we present a brief description of three position-based ad hoc routing protocols that we have chosen to evaluate the scalability. These protocols are the location aided routing (LAR), the distance routing effect algorithm for mobility (DREAM), and the greedy perimeter stateless routing (GPSR). LAR is a reactive position-based ad hoc algorithm. LAR suggested initially to minimize the routing discovery overhead of DSR protocol by an assistant of location position for mobile node [11, 14]. The protocol employees the global position system (GPS) technique, where every node initially can know its area and starts flood the request packets to a restricted area called (Request Zone). RZ defines as a rectangle that holds the source node and the circle of an expected region that contains the last known information of the destination node. This area called (Expected Zone). The intermediate nodes play as an assistant role for forwarding the packets that belong to the same request zone of the sender node. Figure 1 represents the request and expected zones in the LAR protocol.

DREAM is a proactive or on-demand ad hoc protocol [7]. In DREAM every node in the network utilizes up-to-date information about each other node in the network and produces periodically its 
information in the network. Consequently, each node in the network has a complete existence picture to all other nodes. The authors in [7] realized the ideas of "distance effect" and "moving effect" witch is meaning: the further nodes from each other, the slower they appear to be moving, and vice versa. This observation leads - in the end - to optimize the DREAM routing performance by avoiding unnecessary routing overheads.

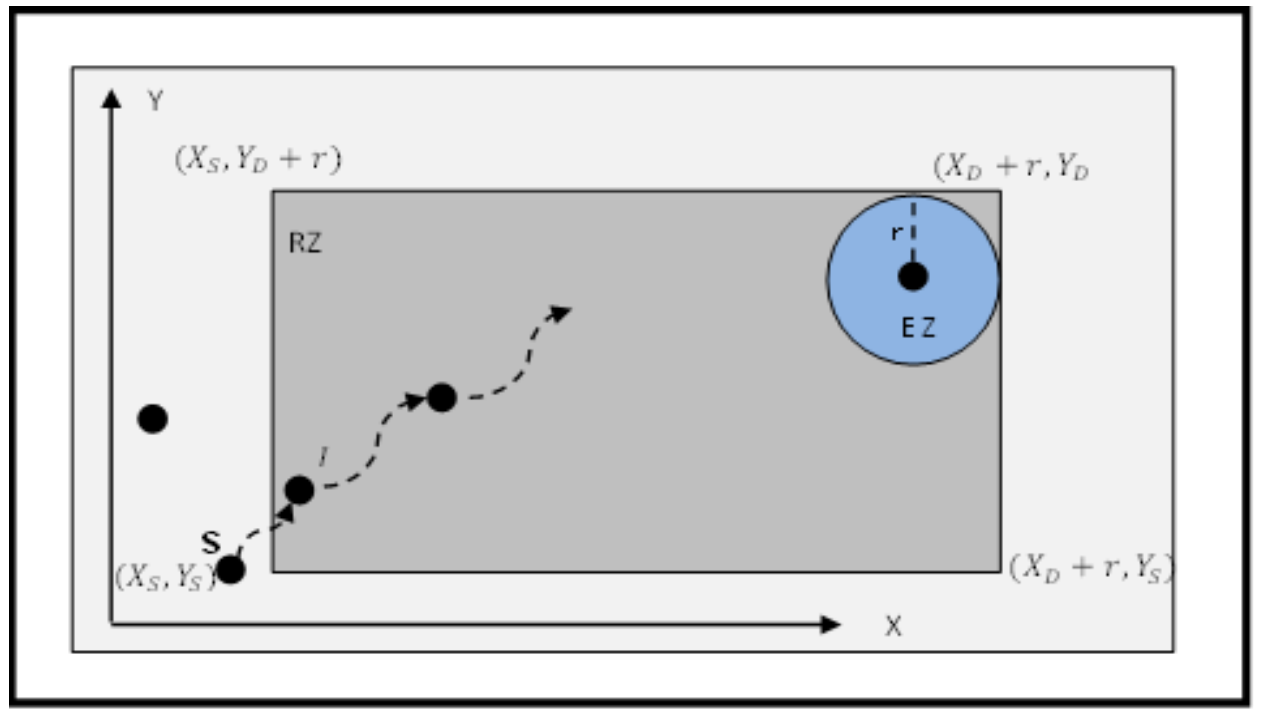

Figure 1. Request zone RZ, expected zone EZ in LAR

The next ad hoc routing protocol of the position-aided type that we're highlighting in this study is the Greedy Perimeter Stateless Routing protocol (GPSR) [21]. This protocol utilizes the local nodes' information where every node periodically sends a 1-hop message called a "beacon" (containing its own identifier and position) which assistance to build a clear picture of its neighbors' positions. In GPSR the sender node forwards the data packet to the neighbor node that has a shorter distance from the target, on another hand; the intermediate node repeats the same process in the direction of the target node as figure out in Figure 2.

GPSR protocol works with two isolated techniques for delivering the communication messages: the greedy mode and the perimeter mode. The packet enters the perimeter mode (recovery mode) when it's arriving at what is known as a "local maximum area", the authors of GPSR implemented the right-hand rule for traversing the graph in order correct the path of traveling packet, on the others side, the packet returns and remains on the greedy mode as long as it has a node closer to destination node from the node where entered the recovery mode.

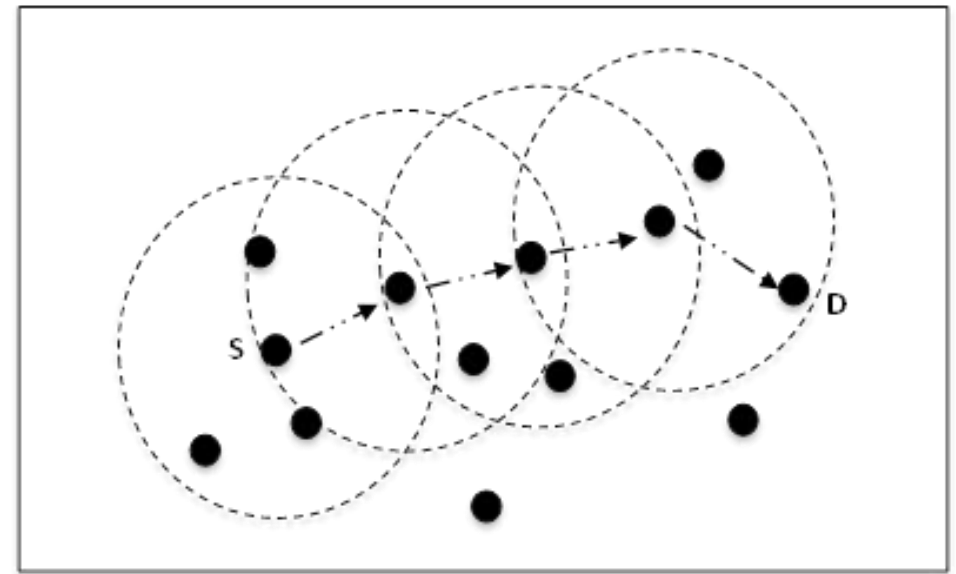

Figure 2. Greedy forwarding data packets in GPSR 


\section{SIMULATION SETUP AND EVALUATION PARAMETERS}

In this study, we evaluate three position-based MANET protocols using the NS2 simulation program (ns-2.35) [31]. Table 3 illustrates the nodes configuration and network topology settings. As we have mentioned in previous sections, in order to evaluate the scalability performance for the routing protocol, we are considering the cases where the network size can be with a fixed amount of nodes (30 nodes) accompanied by four different geographies topology areas $(800 \mathrm{mx} 600 \mathrm{~m}, 1200 \mathrm{mx} 800 \mathrm{~m}, 1600 \mathrm{mx} 1200 \mathrm{~m}$, and $2400 \mathrm{~m} \times 1600 \mathrm{~m})$. Table 3 summarizes these settings.

The mobile nodes are moving according to the Steady-State case of the Random Waypoint model proposed by $[32,33]$. The speed of nodes is uniformly moved in (1 to $20 \mathrm{~m} / \mathrm{s})$ with an average of $5.84 \mathrm{~m} / \mathrm{s}$ and the nodes' pause time is set to $5 \mathrm{~s}$. The wireless transmission range fixed to $250 \mathrm{~m}$ for all mobile nodes. The real traffic payload is produced by Constant Bit Rate application (CBR) in the form of peer-to-peer technique for 10 source nodes sending a data-packed every 250ms (4 packets/s).

Table 3. NS2 parameters and network topology setting

\begin{tabular}{ll}
\hline \multicolumn{1}{c}{ Parameter } & \multicolumn{1}{c}{ Value } \\
\hline Node no. & 30 \\
Mobility model & Random Waypoint \\
Network topology area & $800 \times 600 \mathrm{~m}, 1200 \times 800 \mathrm{~m}, 1600 \times 1200 \mathrm{~m}, 2400 \times 1600 \mathrm{~m}$ \\
Node density $\sim$ & $6.0,2.0,0.8,0.4$ \\
Transmission range & $250 \mathrm{~m}$ \\
Node's speed & $1-20 \mathrm{~m} / \mathrm{s}$ with average of $5.84 \mathrm{~m} / \mathrm{s}$ \\
Node's pause time & $5 \mathrm{~s}$ \\
Simulation time & $300 \mathrm{~s}$ \\
Routing protocols & LAR, DREAM, GPSR \\
Source node & 10 \\
Traffic application & CBR \\
Traffic rate & $250 \mathrm{~ms}(4$ packets $/ \mathrm{s})$ \\
Number of Trials & 5 \\
Medium Access Protocol & IEEE 802.11 \\
\hline
\end{tabular}
metrics are:

We chose four evaluation metrics to compare the performance in scalability factor, these evaluation

a) Hop Count HC: The average of hops that the data packet has leaped in its journey overall data packet arrived at the destination.

b) End -to - End Delay EED: The average time of data packet journey divided by data packet arrived at the destination.

c) Data Packet Delivery Ratio DDR: The ratio of data packets which succeeded to arrive over its destination overall data packets sent.

d) Total Routing Overhead TRO: The summation of all overheads accompanying of routing.

\section{RESULTS AND DISCUSSION}

In this section we first present the results for each single ad hoc protocol with respect to the previously mention parameter metrics (Figures 3, 4, and 5) then we compare results for our three protocols for every single parameter respectively (Figures 6, 7, and 8). The DREAM protocol (Figure 3) has a scientific reflection related to the scalability factor, this appears obviously due to the effect of a pro-active property that has assorted this type of routing protocols, wherein on area sized 2400x1600 EED dropped to 0.0014ms as in Figure 3(a) and the DPDR ratio reduced scientifically to 0.157 as Figure 3(b). While related to LAR protocol (Figure 4) we can point out that the protocol has achieved moderate enhancement in performance evaluation in consort to the scalability issue due to feature of restricted broadcast domain facility that leads to a relative reduction in routing overheads, as we can observe from Figure 4(a) and 4(b) LAR protocol has produced a high ratio of EED and DPDR metrics and can achieve low band in HC and TRO as in Figure 4(c) and 4(d) especially in wide area networks.

In the same context, GPSR protocol (Figure 5) has obtained more enhancing achievements with notes a side effect in simulation processes; this protocol employed the home regional geo-information in order to guarantee data packet delivery, but this comes with more algorithm computational complexity. These observations came compatible with other research studies outcomes that suggested the GPSR protocol as a solution for more stability and reliability of geographically based ad hoc models [34]. 


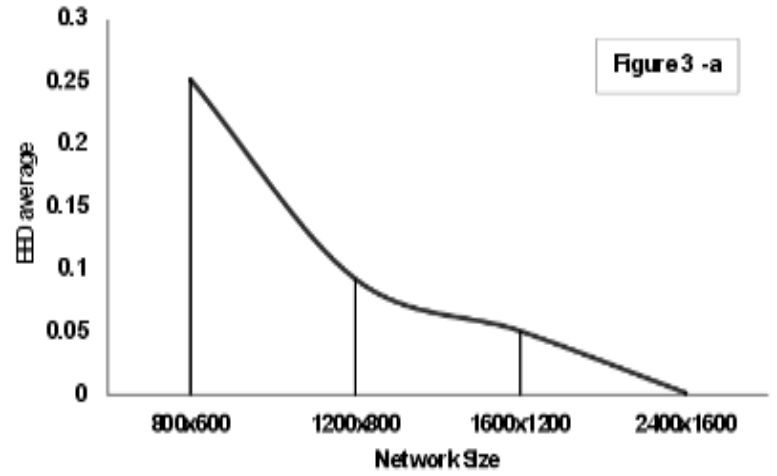

(a)

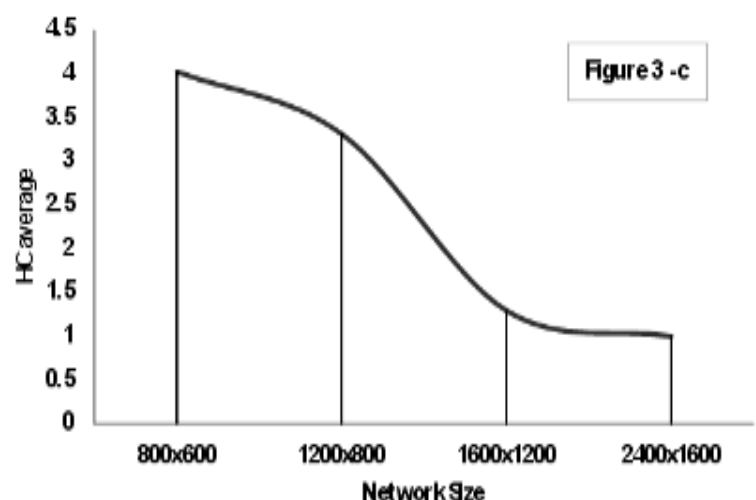

(c)

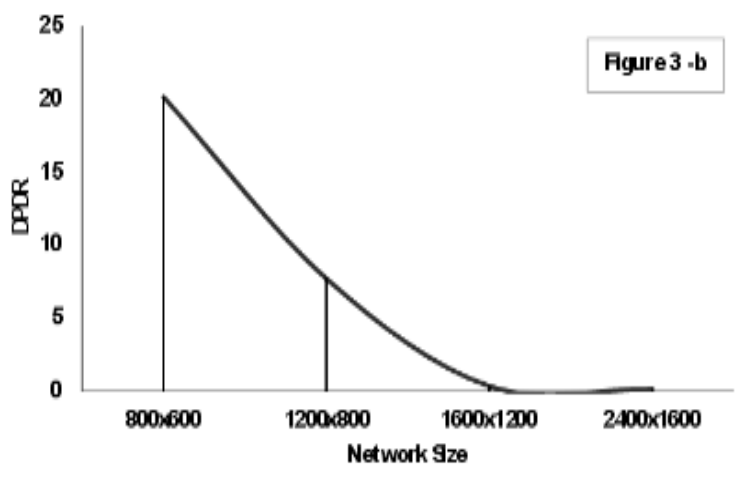

(b)

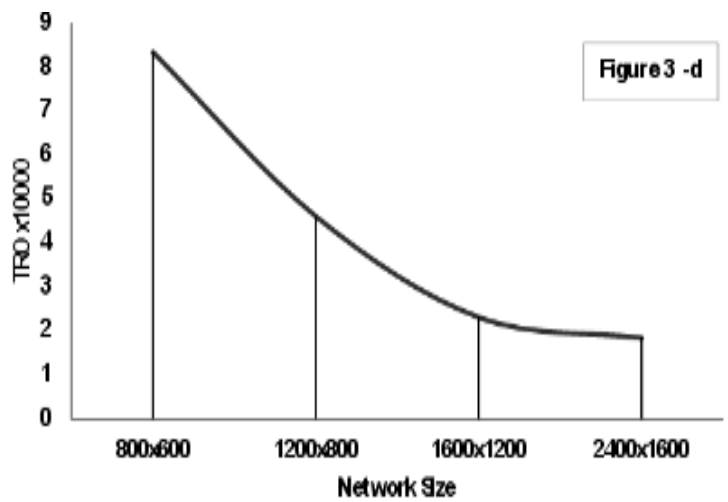

(d)

Figure 3. DREAM with metrics, (a) EED, (b) DPDR, (c) HC, (d) TRO

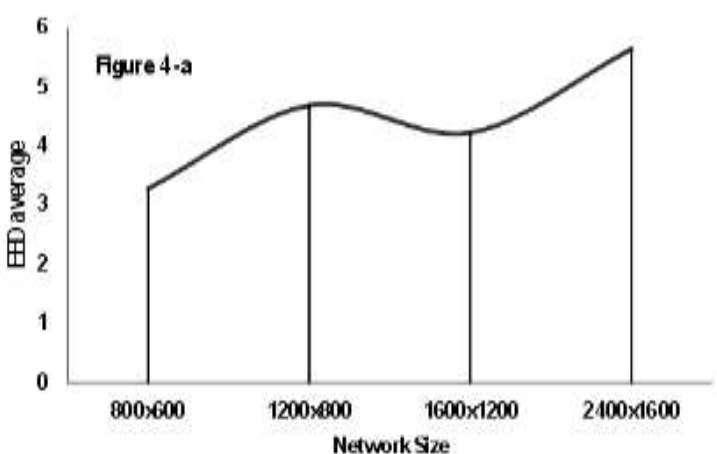

(a)

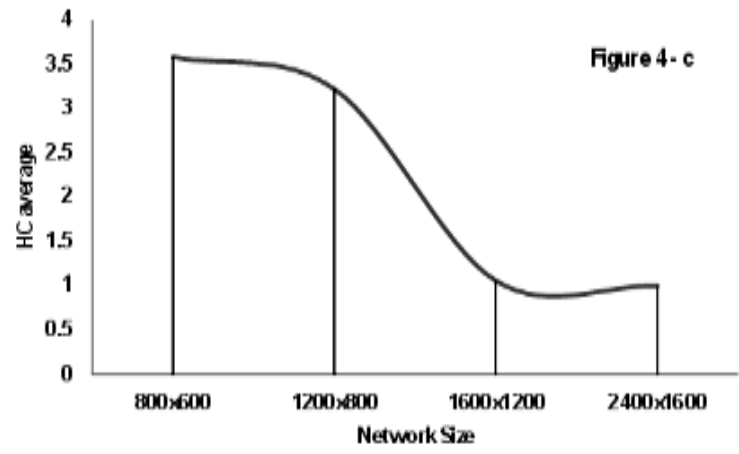

(c)

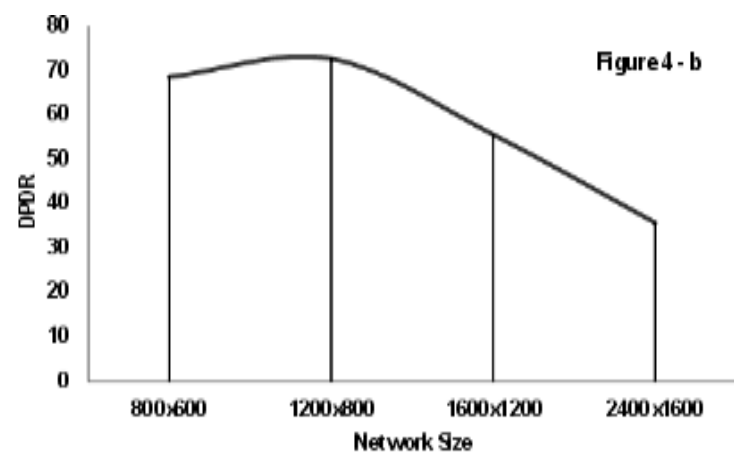

(b)

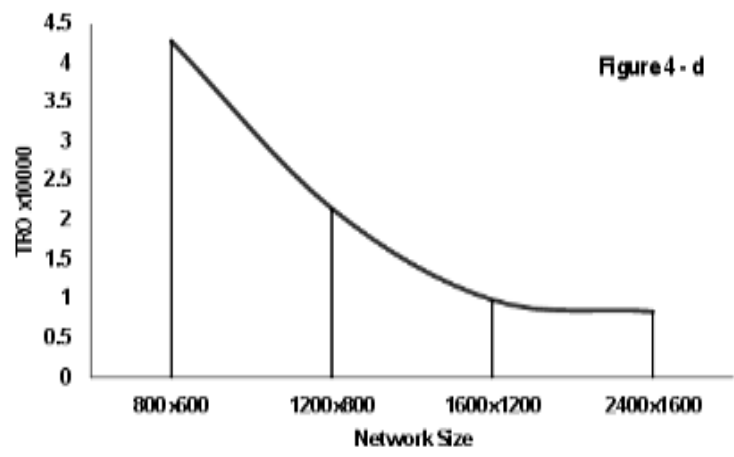

(d)

Figure 4. LAR with metrics, (a) EED, (b) DPDR, (c) HC, (d) TRO 


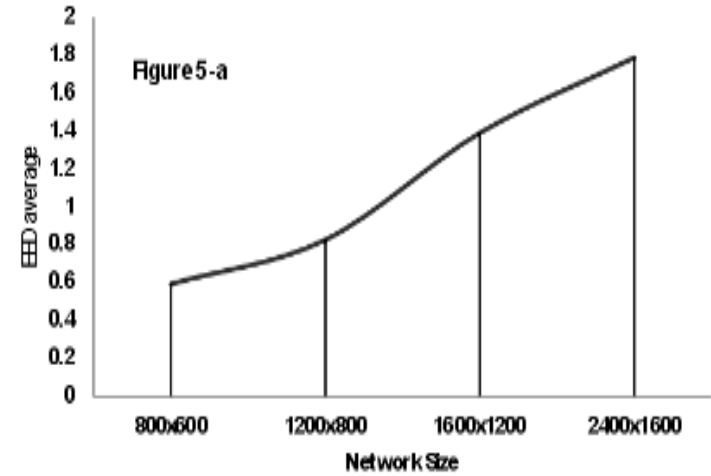

(a)

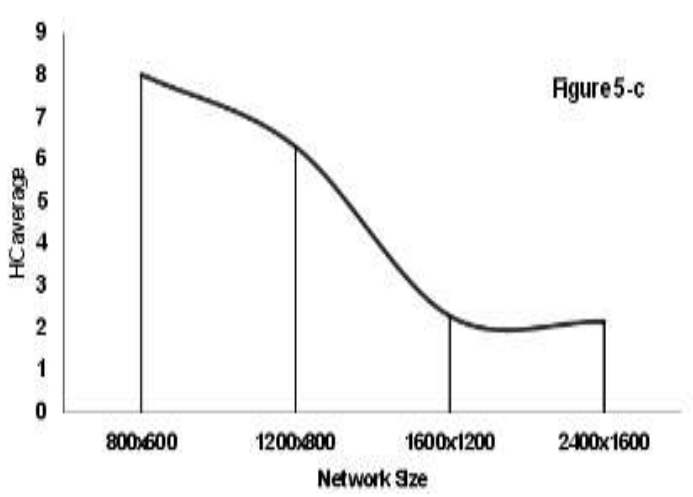

(c)

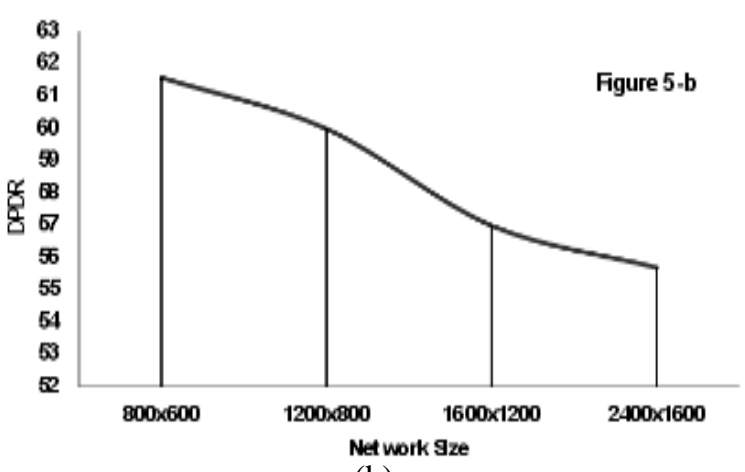

(b)

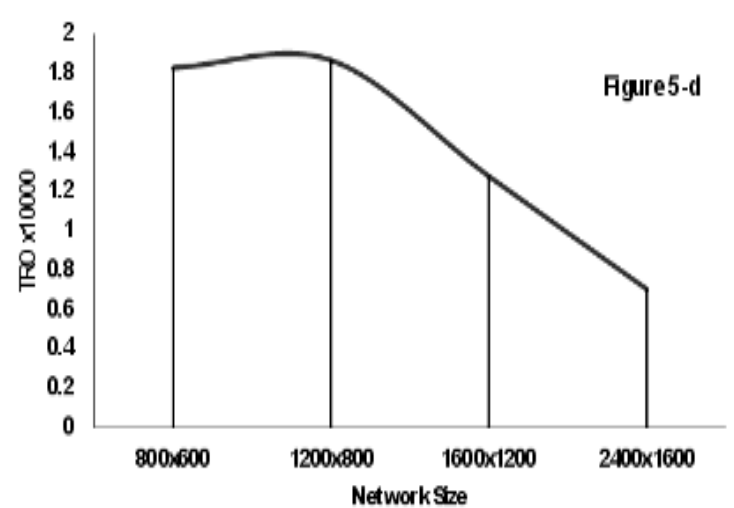

(d)

Figure 5. GPSR with metrics, (a) EED, (b) DPDR, (c) HC, (d) TRO

Figures 6 to 9 represent the comparison between DRAM, LAR, and GPSR protocols with respect to the four evaluation parameters (EED, DPDR, HC, AND TRO) respectively. in Figure 6 we can point out that the delay times for sending data packets in DREAM and GPSR protocols have obtained fine results, especially in DREAM due to the reliability coming from the global style of pro-active design, while, LAR protocol has lacked to achieve.

Figure 7 represents the simulation result of the data delivery ratio. We can observe that the GPSR protocol has achieved the stability ratio to deliver data along the line of network size changing, while the result in Figure 8 comes with diverse variations regarding the number of vesting hops during the data packet's journey. Figure 9 illustrates that GPSR protocol has got an enhancement in performance function to tolerance network size-changing which exceeds other position-based routing protocols, this comes clearly regarding computing the routing overheads metric.

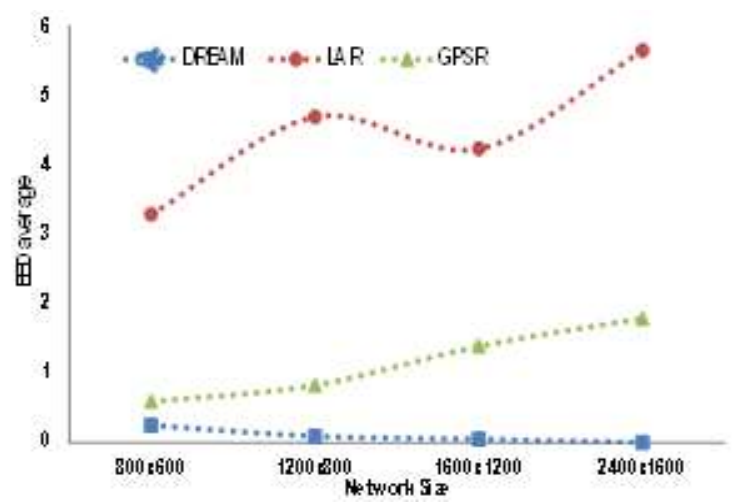

Figure 6. End-to-end delay time

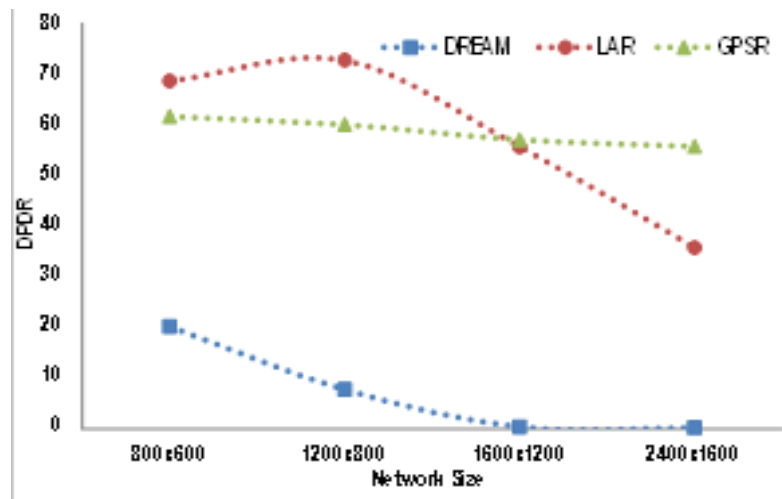

Figure 7. Data packet delivery ratio 


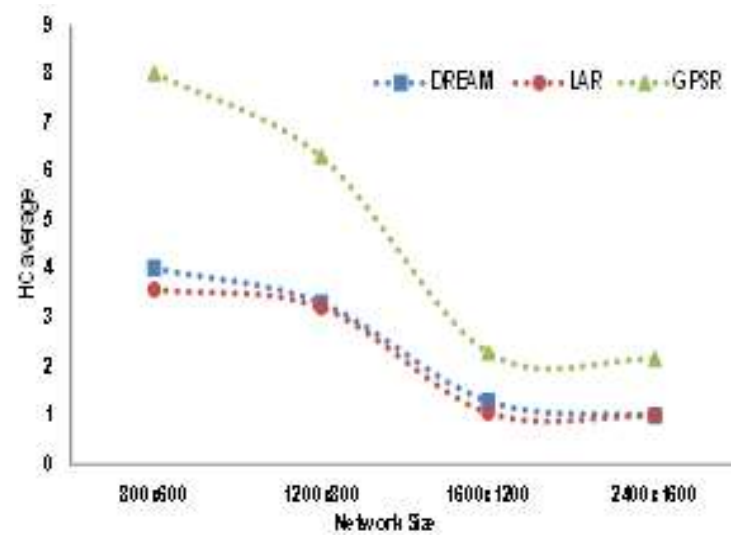

Figure 8. Average hop count

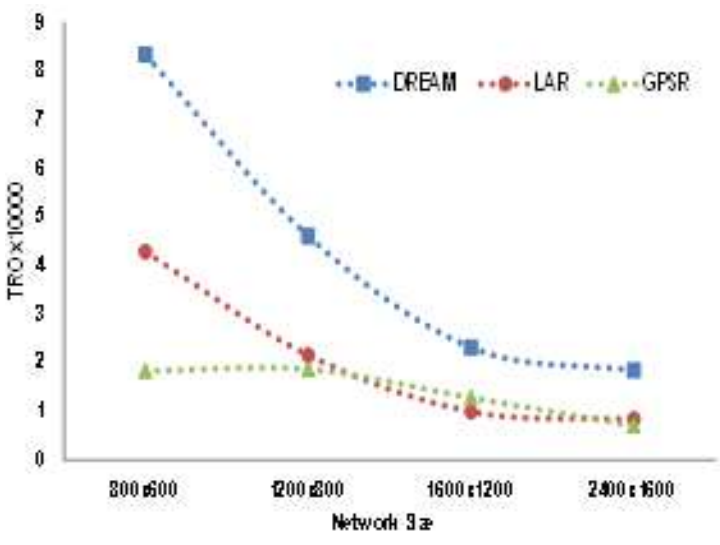

Figure 9. Total routing overhead

\section{CONCLUSIONS}

The scalability subject plays a scientific feature in position-based ad hoc routing protocols. In this paper, we evaluated the performance of three geographical aided ad hoc algorithms in order to study the capability of changing network size while remaining the volume of nodes constant. We observed that the proactive type protocols like DREAM protocol can be suitable with a minimum small area of networks when the threshold of delay will be considered and the energy of nodes is scarce. On another hand, the local geoinformation service in greedy type's protocols like GPSR assist the mobile nodes to guarantee to deliver its data packets in spite of network size changing, however, this came with the expensive of computational complexity, on another side, LAR protocol has considered as moderate performance related to the scalability problem. For future works, we suggest taking the factor of node's energy in the performance consideration.

\section{ACKNOWLEDGEMENTS}

The authors are very grateful to the University of Mosul, College of Computer Sciences and Mathematics for their provided facilities, which helped to improve the quality of this work.

\section{REFERENCES}

[1] N. Raza, et al., "Mobile ad-hoc networks applications and its challenges," Communications and Network, vol. 8, no. 3, pp. 131-136, 2016.

[2] M. Mauve, et al., "A survey on position-based routing in mobile ad hoc networks," IEEE network, vol. 15, no. 6, pp. 30-9, 2001.

[3] T. H. Clausen, et al., "Optimized LinkState Routing Protocol (OLSR)," Network Working Grup, 2003.

[4] C. A. Santiváñez, et al., "Making link-state routing scale for ad hoc networks," in Proceedings of the 2nd ACM international symposium on Mobile ad hoc networking \& computing, pp. 22-32, 2001.

[5] C. E. Perkins and P. Bhagwat, "Highly dynamic destination-sequenced distance-vector routing (DSDV) for mobile computers," ACM SIGCOMM computer communication review, vol. 24, no. 4, pp. 234-44, 1994.

[6] R. G. Ogier, "Topology broadcast based on reverse-path forwarding (TBRPF)," Internet Engineering Task Force (IETF) draft, 2002.

[7] S. Basagni, et al., "A distance routing effect algorithm for mobility (DREAM)," in Proceedings of the 4th annual ACM/IEEE international conference on Mobile computing and networking, pp. 76-84, 1998.

[8] C. H. Chou, et al., "Dynamic route maintenance for geographic forwarding in mobile ad hoc networks," Computer Networks, vol. 52, no. 2, pp. 418-31, 2008.

[9] Y. Liu, et al., "A region-based routing protocol for wireless mobile ad hoc networks," IEEE network, vol. 18, no. 4, pp. 12-7, 2004.

[10] D. B. Johnson and D. A. Maltz, "Dynamic source routing in ad hoc wireless networks," in Mobile computing, pp. 153-181, 1996.

[11] C. Perkins, et al., "RFC3561: Ad hoc on-demand distance vector (AODV) routing," Network Working Grup, 2003.

[12] A. N. Le, et al., "Load-aware routing protocol for multi-radio wireless mesh networks," in 2008 Second International Conference on Communications and Electronics, pp. 138-143, 2008.

[13] V. D. Park and M. S. Corson, "A highly adaptive distributed routing algorithm for mobile wireless networks," in Proceedings of INFOCOM'97, vol. 3, pp. 1405-1413, 1997.

[14] Y. B. Ko and N. H. Vaidya, "Location-Aided Routing (LAR) in mobile ad hoc networks," Wireless networks, vol. 6 , no. 4, pp. 307-21, 2000. 
[15] Y. B. Ko and N. H. Vaidya, "GeoTORA: A protocol for geocasting in mobile ad hoc networks," in Proceedings 2000 international conference on network protocols, pp. 240-250, 2000.

[16] K. El Defrawy and G. Tsudik, "ALARM: Anonymous location-aided routing in suspicious MANETs," IEEE Transactions on Mobile Computing, vol. 10, no. 9, pp. 1345-1358, 2010.

[17] M. R. Pearlman and Z. J. Haas, "Determining the optimal configuration for the zone routing protocol," IEEE Journal on Selected Areas in Communications, vol. 17, no. 8, pp. 1395-414, 1999.

[18] M. Palaniammal and M. Lalli, "Comparative study of routing Protocols for MANETs," International Journal of Computer Science and Mobile Applications, vol. 2, no. 2, pp. 118-127, 2014.

[19] G. Koltsidas, et al., "A performance study of the hsls routing algorithm for ad hoc networks," in 2004 IEEE 59th Vehicular Technology Conference. VTC 2004-Spring, vol. 4, pp. 2140-2143, 2004.

[20] T. Hamma, et al., "An efficient zhls routing protocol for mobile ad hoc networks," in 17th International Workshop on Database and Expert Systems Applications (DEXA'06), pp. 66-70, 2006.

[21] B. Karp and H. T. Kung, "GPSR: Greedy perimeter stateless routing for wireless networks," in Proceedings of the 6th annual international conference on Mobile computing and networking, pp. 243-254, 2000.

[22] V. C. Giruka and M. Singhal, "A self-healing on-demand geographic path routing protocol for mobile ad-hoc networks," Ad Hoc Networks, vol. 5, no. 7, pp. 1113-1128, 2007.

[23] E. Belding-Royer, "Routing approaches in mobile ad hoc networks," in S. Basagni, et al. (eds), Ad Hoc Networking, IEEE Press Wiley, New York, 2003.

[24] M. Käsemann and H. Hartenstein, "Analysis of a location service for position-based routing in mobile ad hoc networks," in Mobile Ad-Hoc Netzwerke, 1 deutscher Workshop über Mobile Ad-Hoc Netzwerke WMAN, 2002.

[25] X. Hong, et al., "Scalable routing protocols for mobile ad hoc networks," IEEE network, vol. 16, no. 4, pp. 11-21, 2002.

[26] P. Yao, et al., "Performance comparison of geocast routing protocols for a MANET," in Proceedings of 13th International Conference on Computer Communications and Networks, pp. 213-220, 2004

[27] D. O. Jorg, "Performance comparison of MANET routing protocols in different network sizes," University of Berne, Switzerland, 2003

[28] L. Blazevic, et al., "A location-based routing method for mobile ad hoc networks," IEEE Transactions on mobile computing, vol. 4, no. 2, pp. 97-110, 2005.

[29] M. Bakhouya and N. Cottin, "Performance evaluation of the location-based protocol dream for large mobile ad hoc networks," in 2008 New Technologies, Mobility and Security, pp. 1-6, 2008.

[30] C. Walsh, et al., "A call to arms: it's time for REAL mobility models," ACM SIGMOBILE Mobile Computing and Communications Review, vol. 12, no. 1, pp. 34-6, 2008.

[31] https://www.isi.edu/nsnam/ns/

[32] W. Navidi, et al., 'Improving the accuracy of random waypoint simulations through steady-state initialization," in Proceedings of the 15th International Conference on Modeling and Simulation, pp. 319-326, 2004.

[33] R. B. Mahmood, "Impact of Mobility Models on Performance of Ad hoc Position based Routing Protocol LAR," International Journal of Computer Applications, vol. 181, no. 40, pp. 27-31, 2018

[34] Y. Al-Roqi, et al., "Performance evaluation of three GPSR-based routing protocols in a military setting," in Information, Intelligence, System and Applications (IISA 2013), pp. 1-4, 2013. 